\title{
Quantitative simulation of silica scale deposition from physical kinematics perspectives
}

\author{
Masaki IWATA $^{1}$, Hitoshi MIKADA ${ }^{1}$ and Junichi TAKEKAWA ${ }^{1}$ \\ ${ }^{1}$ Dept. of Civil and Earth Res. Eng., Kyoto University
}

\begin{abstract}
Silica scaling restricts the heat extraction and deteriorates the power generation efficiency in geothermal systems. We conducted a deeply stepped analysis on the scaling phenomena with physical kinematics. We simulated the mechanical action on fine particles considered to be spherical in geothermal fluid. In addition, we evaluated the probability of particle re-entrainment from the wall surface and compared the scale deposition rate obtained from different ways of direct calculations. We succeeded not only in matching the order of deposition rate with experimental data, but also explaining the tendency of increase in partial scale deposition amount. Furthermore, our simulation using particles with various diameters indicates the prevention effect of scale buildup by controlling the colloidal aggregation.
\end{abstract}

\section{INTRODUCTION}

Scale deposition can damage the pipe systems in geothermal power plants. Although the formation of scale on the pipe wall due to produced mineral salts could be mitigated with chemical treatments or surface modifications, these countermeasures are still at an insufficient level. Siliceous scale deposition most often occurs when the geothermal brine initially at or near saturation in reservoirs is cooled and supersaturated in the course of energy extraction. Accurate predictions of this phenomenon can give way to preventive maintenance for sustainable energy resources. Hence, the reliable and quantitative simulation of the scale accumulation is critical for production availability.

As a novel initiative which is completely different from the chemical prediction method of scale growth widely used so far, Mizushima et al. (2016) proposed a scale precipitation prediction method that took physical factors acting on silica particles into account, and reproduced the increase of local scale deposition depending on fluid flow velocity $y^{1,2}$. Simplified and omitted elements in this approach have been complemented by another study which is also based on physical kinematics ${ }^{3)}$.

In this research, we further investigate the theories of hydrokinetics to deal with scale accumulation process and carry out a more detailed calculation with respect to the effect of fluid shear rate and stochastic elements on particle motion. Besides the computation of uniform particles moving from silica suspension to the wall surface, we put forward the quantitative and qualitative simulation of fine particles on the wall where some particles come unstuck with the aim of elucidating the field observation $^{4)}$ and scaling diversity correlated with polymerization of the silica monomer.

\section{SIMULATION MODEL}

\section{(1) Particle behavior in colloidal suspension}

We regard the silica involved in scale deposition as spherical colloidal particles with the diameter of $100 \mathrm{~nm}$ and evaluate the mechanical balance in the process where the silica particles flowing in the medium fluid adhere to the wall surface.

\section{a) Fluid condition}

We set a two-dimensional parallel plate channel (Figure 1) in order to reproduce the geothermal fluid flow. The current of hot water inside the piping system is regarded as the Hagen-Poiseuille flow and the properties of geothermal fluid including flow rate, temperature, wall material and pipe diameter are the same as those of laboratory experiment ${ }^{5)}$ conducted by Hosoi and Imai (1982) for the validation of our calculation. Concerning the fluid behavior near the wall surface, we use two types of boundary condition with and without slip flow ${ }^{6}$.

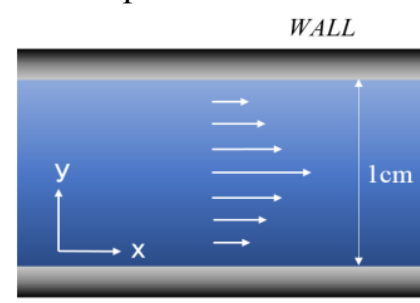

WALL

Figure 12D parallel plate channel 


\section{b) Motion equation}

We directly calculated discretized equation of particle motion which is described as

$$
m \dot{\boldsymbol{u}}_{p}=\boldsymbol{F}_{D}+\boldsymbol{F}_{B G}+\boldsymbol{F}_{V d W}+\boldsymbol{F}_{E L E}+\boldsymbol{F}_{B}
$$

where $m$ is particle mass, $\dot{\boldsymbol{u}}_{p}$ is particle acceleration vector, $\boldsymbol{F}_{D}$ is drag force by fluid, $\boldsymbol{F}_{B G}$ is the total of gravity and buoyancy force, $\boldsymbol{F}_{V d W}$ is the Van der Waals attractive force, $\boldsymbol{F}_{E L E}$ is electrostatic repulsive force, and $\boldsymbol{F}_{B}$ is force by the Brownian motion. With this direct numerical analysis, it is possible to track the particle behavior stably without any restriction due to the grid intervals.

\section{c) Particle adhesion to the wall}

In the process of particle adhesion to the wall surface, we use the simulation method ${ }^{3)}$ based on diffusion behavior due to the fact that the dominant factor for the particle motion with the particle size below $500 \mathrm{~nm}$ is the Brownian motion ${ }^{7}$. Figure 2 describes one of the particle trajectories.

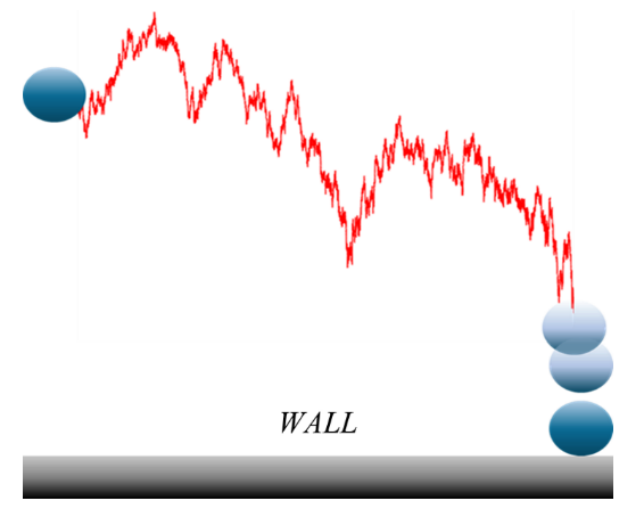

Figure 2 Diffusion orbit of a colloidal particle

(2) Particle re-entrainment from the wall surface

There is a field observation that clarifies relatively large amount of scale deposition in the stagnant part of the flow ${ }^{4}$ (Figure 3). As a process incorporating the influence of shear flow, we adopt the rotarydetachment model ${ }^{3,8-12}$. Figure 4 shows a schematic figure of the rotational moment balance around the contact part $P$, where $d$ is particle diameter, $a$ is contact radius obtained from the Hertz's elastic contact theory ${ }^{13)}$. The particle detachment condition by taking moment balance into consideration is expressed as

$$
\boldsymbol{F}_{S} \cdot \sqrt{\left(\frac{d}{2}\right)^{2}-a^{2}}>\boldsymbol{F}_{A} \cdot a
$$

where $\boldsymbol{F}_{S}$ is separation force mainly composed of hydrodynamic action and $\boldsymbol{F}_{A}$ is adhesion force.

\section{a) Stochastic process by random force}

Assuming that the Brownian random force acts on the uniform particles on the wall surface as well as in the fluid, we add $\boldsymbol{F}_{B}$ to $\boldsymbol{F}_{S}$ and $\boldsymbol{F}_{A}$. Of the 100,000 particles, the number of particles satisfying the condition (2) is counted and incorporated into scale deposition rate.

\section{b) Stochastic process by particle size distribution}

It has been reported that the Brownian motion of particles may have anisotropy near the interface ${ }^{14,15)}$. In consideration of this possibility, we introduce a stochastic process with another approach that gives variation in particle size. As the first step for this simulation, we analyzed the critical diameter of silica particle for the shear flow (Figure 4). The threshold at which the separation moment exceeds the adsorption moment and the particles peel off seems to vary significantly depending on the properties of the fluid flow. Regarding the distribution function of particle diameter, it has been reported that particles in a colloidal solution form a bimodal distribution since colloidal particles suspended in water in the range from several $\mathrm{nm}$ to $\mu \mathrm{m}$ agglomerate with or repel each other according to the surrounding conditions ${ }^{16,17)}$ (Figure 5). We also apply this functional model to the calculation of particle detachment rate and conduct a more multifaceted analysis of the effect of shear flow on scale growth. The probability density function of particle number $N$ is described in the form of a lognormal distribution as

$$
\begin{aligned}
\frac{d N}{d \ln r} & =\frac{N_{f}}{\sqrt{2 \pi} \ln \sigma_{f}} \exp \left[-\frac{\left(\ln r-\ln r_{f}\right)^{2}}{2\left(\ln \sigma_{f}\right)^{2}}\right] \\
& +\frac{N_{c}}{\sqrt{2 \pi} \ln \sigma_{c}} \exp \left[-\frac{\left(\ln r-\ln r_{c}\right)^{2}}{2\left(\ln \sigma_{c}\right)^{2}}\right]
\end{aligned}
$$

where $N_{f}, \sigma_{f}, r_{f}, N_{c}, \sigma_{c}$ and $r_{c}$ are modal particle number, standard deviation and modal radius of fine and coarse particles, respectively. As a parameter that determines the shape of each mode, the volume ratio of fine modal particles $f$ is represented by

$$
f=\frac{V_{f}}{V_{f}+V_{c}}
$$

where $V_{f}$ and $V_{c}$ are the volume of fine and coarse particles. By integrating the probability density function, we obtain the total weight of particles that don't move from the wall under specific conditions 
and incorporate it into the particle detachment rate. Here, we set the mean diameter of colloidal particle to $100 \mathrm{~nm}$ and the volume ratio $f$ to $0.20,0.25,0.50$, 0.75 , and 0.80 .

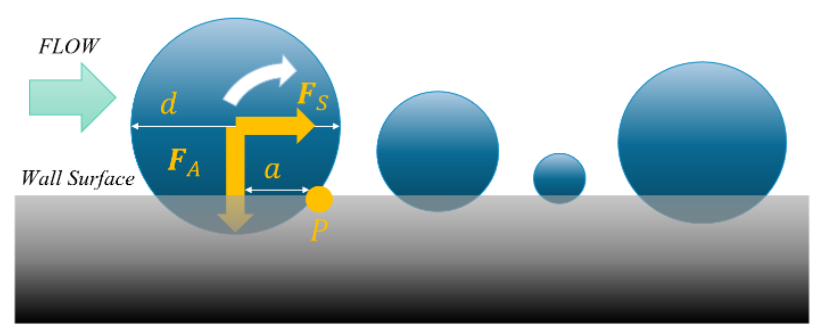

Figure 3 Rotary-detachment model

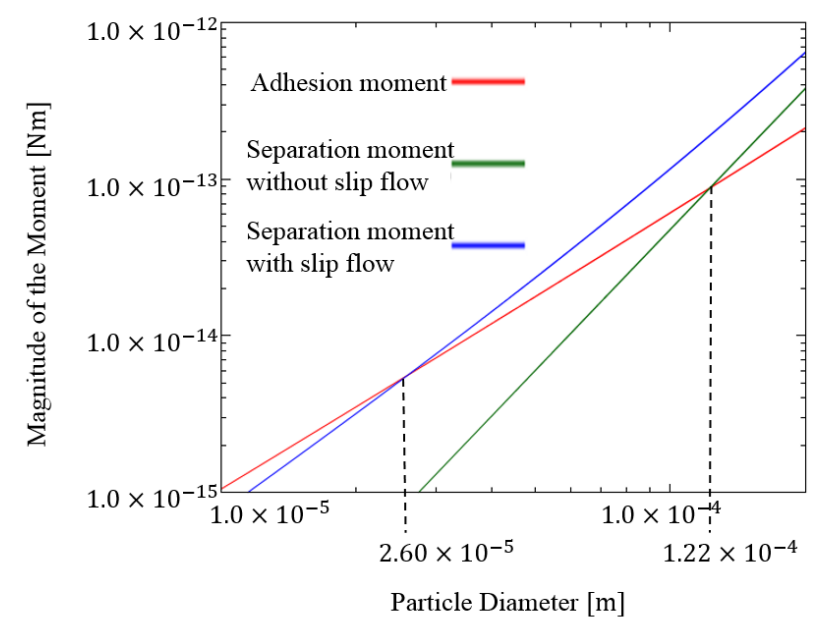

Figure 4 Comparison of magnitude of each moment in Rotary-detachment model without Brownian motion

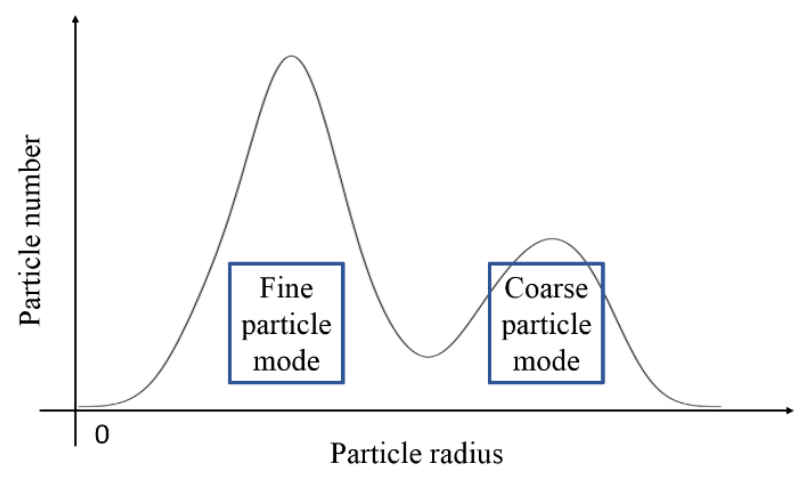

Figure 5 Bimodal distribution function

\section{RESULTS}

\section{(1) Particle detachment rate}

Figure 6 shows the change of each detachment rate of particles where the shear velocity of medium fluid is in the realistic range. In the case where particles have variations in diameter, the particle detachment rate is more sensitive than previous simulation with Brownian motion and it is expected that the rate ultimately converges as the shear velocity increases. Each result could give a consistent explanation to the field observation ${ }^{4)}$.

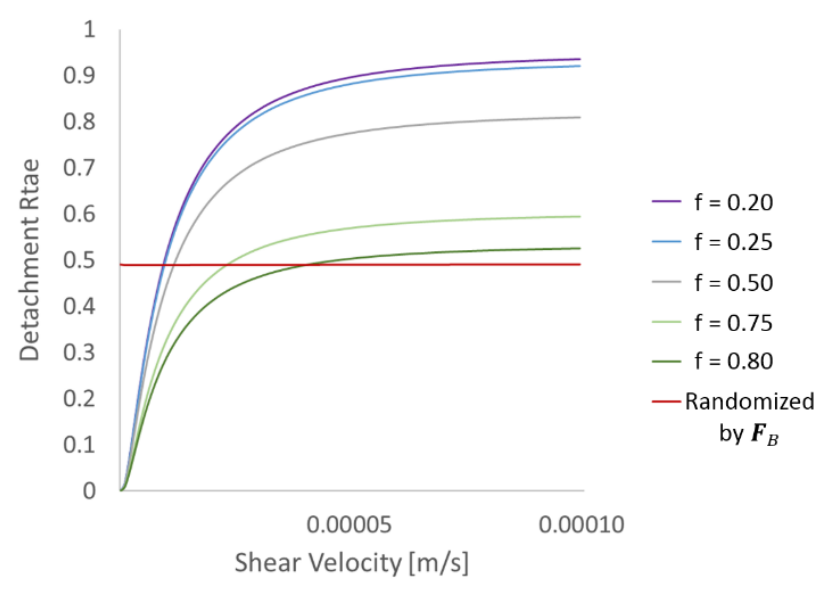

Figure 6 The relationship between fluid shear velocity and particle detachment rate

\section{(2) Quantitative validation of scale deposition}

Table 1 shows the amount of scale accumulation on the pipe wall per unit surface area. Our calculation results show good agreement with the experimental data $^{5)}$ and suggest that the scale deposition can be suppressed by accelerated polymerization of the colloidal particles.

Table 1 Scale deposition amount per $24 \mathrm{~h}$

\begin{tabular}{|c|c|c|c|}
\hline & \multicolumn{3}{|c|}{ Scale precipitation $\left[\mathrm{g} / \mathrm{m}^{2}\right]$} \\
\hline & $f$ & $\begin{array}{c}\text { No-slip } \\
\text { Flow }\end{array}$ & $\begin{array}{l}\text { Slip } \\
\text { Flow }\end{array}$ \\
\hline \multirow{6}{*}{$\begin{array}{l}\text { Simulation } \\
\text { results }\end{array}$} & 0.20 & 1.1 & 0.69 \\
\hline & 0.25 & 1.1 & 0.70 \\
\hline & 0.50 & 1.1 & 0.74 \\
\hline & 0.75 & 1.1 & 0.84 \\
\hline & 0.80 & 1.1 & 0.87 \\
\hline & $\begin{array}{c}\text { Randomized } \\
\text { by } F_{B}\end{array}$ & 0.56 & 0.55 \\
\hline \multicolumn{2}{|c|}{ Experimental data $^{5}$} & \multicolumn{2}{|c|}{$0.42 \sim 1.7$} \\
\hline
\end{tabular}

\section{CONCLUSION}

We developed the hydrodynamic approach of scale deposition and constructed a quantitative method covering a broad range of actual phenomena with random elements. Numerical result of silica deposition rate is in good consistency with that of the measured data in laboratory and geothermal field. It is a remarkable achievement in this research that we 
elucidate the necessity to incorporate the physical process into the prediction of scale deposition and the possibility of scale inhibition with particle polymerization.

\section{REFERENCES}

1) Mizushima, A., Mikada, H. and Takekawa, J., 2015, The Importance of Hydrodynamic Conditions in Silicate Scale Growth Inferred From Numberical Simulation, Transactions Geothermal Resources Council, 39, 489-494.

2) Mizushima, A., Mikada, H. and Takekawa, J., 2016, The role of physical and chemical processes of silica scale growth in geothermal wells, Recent Advances in Exploration Geophysics (RAEG 2016), doi: 10.3997/23528265.20140209.

3) Iwata, M., Mikada, H. and Takekawa, J., 2017, Kinematics of a silica particle in the deposition to piping of geothermal power plant, 79th EAGE Conference and Exhibition 2017, accepted.

4) Mercado, M., F. Bermejo, R. Hurtado, B. Terrazas, and L. Hernandez, 1989, Scale incidence of production pipes of Cerro Prieto geothermal wells, Geothermics, 18, 225-232.

5) Hosoi, M. and Imai, H., 1982, Study on precipitation and prevention of the silica scale from the geothermal hot water, Journal of the Geothermal Research Society of Japan, 4, 127142.

6) Kojima, N., 1988, On the Unified Expression of Liquid Slippage on the Pipe Wall under the Laminar Flow.

7) Theodore, L., and Kunz, R. G., 2005, Nanotechnology: environmental implications and solutions. John Wiley \& Sons.

8) Matsusaka, S., Koumura, M., and Masuda, H., 1997, Analysis of adhesive force between particle and wall based on particle reentrainment by airflow and centrifugal separation, Kagaku Kogaku Ronbunshu, 23(4), 561-568.

9) Jimbo,G., Yamazaki, R. and Hong, G., 1981, The Development of New Measuring Methods of Particle and Powder Bed, Rep. Asahi Glass Found. Ind. Technol., 38, 123-134.

10) Kousaka, Y., Okuyama, K., and Endo, Y., 1980, Re-entrainment of small aggregate particles from a plane surface by air stream, Journal of Chemical Engineering of Japan, 13(2), 143-147.

11) Wang, H. C., 1990, Effects of inceptive motion on particle detachment from surfaces. Aerosol Science and Technology, 13(3), 386-393.

12) Tsai, C. J., Pui, D. Y., and Liu, B. Y., 1991, Particle detachment from disk surfaces of computer disk drives. Journal of aerosol science, 22(6), 737-746.
13) Timoshenko, S., and Goodier, J. N., 1951, Theory of elasticity., New York, 412, 108.

14) Hosoda, M., Sakai, K. and Takagi, K., 1998, Measurement of anisotropic Brownian motion near an interface by evanescent light-scattering spectroscopy, Phys. Rev. E, 58, 6275-6280.

15) Brenner, H., 1961, The slow motion of a sphere through a viscous fluid towards a plane surface, Chem. Eng. Sci., 16, 242-252.

16) Mok, K. R. C., Jaraiz, M., Martin-Bragado, I., Rubio, J. E., Castrillo, P., Pinacho, R., Srinivasan, M. P., Benistant, F., 2005, Materials Science and Engineering B-Solid State Materials for Advanced Technology, 124, 389.

17) Dukhin, A., Goetz, P., 2005, Colloids and Surfaces a-Physicochemical and Engineering Aspects, 253, 51 . 